\section{Subproblem analysis of discrimination shift learning in the rat*}

\author{
THOMAS J. TIGHE and KARL FREY \\ Dartmouth College, Hanover, N.H. 03755
}

Rats were given reversal and extradimensional shifts in a simultaneous discrimination under three conditions designed to vary the likelihood that Ss process the constituent stimulus pairs (two) as instances of a single problem. Condition I involved conventional training whereby the stimulus pairs were presented successively in random order. Condition II was the same as I except that $S$ responded to the two pairs in temporally and spatially defined runs. In Condition III, the pairs were presented as in II but $\mathbf{S}$ was reinforced throughout training only if there had been correct choice to both pairs in the run. Analysis of learning on the individual stimulus pairs during shifts indicated that under all conditions, Ss learned the pairs as independent subproblems.

Tighe, Glick, \& Cole (1971) have recently suggested an analysis of discrimination shift performance that emphasizes the importance of pattern of learning rather than ease of learning as in conventional analyses. They argue that the stimulus instances of a concept-shift task can be viewed as subproblems that may be learned more or less independently of one another despite the fact that they vary on the same dimensions; and they point out that the nature of subproblem learning can be tested in extradimensional (ED) shifts that employ the same stimuli as in preshift training. Under this procedure, one-half of the task instances maintain the stimulus-reward relations that prevailed in the preshift training, while the other half involve a reversal of stimulus-rew ard relations. If Ss do learn the task instances independently, only those instances that undergo change in reward relations should pose substantial relearning problems. Independence in subproblem learning, then, can be measured in terms of the difference between the shift learning functions exhibited on the changed and unchanged instances. Moreover, the analysis indicates that independent subproblem learning should be correlated with faster ED than reversal (R) shift, since the former involves fewer changed instances than the latter. The relative ease with which organisms accomplish $\mathrm{R}$ vs ED shifts has been a matter of considerable interest to theories of discrimination learning (Kendler, 1971).

Tighe et al (1971) applied the subproblem analysis to a two-dimension, two-pair R-ED comparison in children. They found

* This research was supported by Grant $\mathrm{MH}-1 \mathbf{1 0 8 8}$ from the National Institutes of Health. Thomas Tighe is supported by Research Career Development Award K4-HD-43,859 from the National Institute of Child Health and Human Development. Thanks are due $R$. Leaton for valuable comments on the manuscript. that 10-year-olds exhibited similar learning functions on unchanged and changed pairs of ED shift and high error rates on both pairs. In contrast, 4-year-olds made relatively few errors on the unchanged stimulus pair of ED shift but showed a gradual reversal-like acquisition of correct response to the changed pair. Graf \& Tighe (1971) found even more marked evidence of independence in subproblem learning in turtles trained in similarly structured tasks. In an ED shift these Ss maintained errorless performance on the unchanged stimulus pair throughout relearning of the reversed pair. In these experiments, independence in subproblem learning was correlated with fast ED shift.

The aims of the present experiment were, first, to extend the subproblem analysis to other infrahuman species, and second, to attempt to alter the pattern of subproblem learning. The first aim is embodied in Condition I of the experiment that provided, in major respects, a replication of Kelleher's (1956) study of $R$ and ED shifts in rats within a two-dimensional, two-pair discrimination. Kelleher's task was selected since he reported faster $E D$ than $R$ shift under conditions appropriate to application of the subproblem analysis. By the previous assumptions, then, independence in subproblem learning was expected to obtain under Condition I.

Reduction in independence was sought in Conditions II and III, which were designed to increase the likelihood that Ss process the stimulus pairs as instances of a single problem. Condition II was identical to Condition I except that $S$ responded to the two pairs in temporally and spatially defined "runs" with reinforcement following choice to each pair. This procedure was adopted in view of Leary's observation (1962) that monkeys tended to generalize reversal behavior across unrelated stimulus pairs when these were presented within a list structure. In Condition III, the pairs were presented as in Condition II, but $S$ was reinforced only if there had been correct choice to both pairs in the run. This training makes reinforcement (and mastery of the problem) contingent upon occurrence of a common response across the constituent pairs.

\section{SUBJECTS}

The Ss were 40 experimentally naive 90-day-old male albino rats from the Holtzman, Wisconsin, supply. The Ss were maintained at $80 \%$ to $85 \%$ of their free-feeding weights throughout training.

APPARATUS
The apparatus consisted of two duplicate discrimination training units connected end to end. These units were constructed of $1 / 2$-in. plywood, and each consisted of a $9 \times 9 \times 6$ in. startbox that opened onto two of three $4 \times 22 \times 6$ in. parallel adjacent alleys. Guillotine doors of unpainted Masonite were placed at the entrance of each alley of the first unit and separated the start and alley sections of that unit. Similar doors placed at the end of each alley in the first unit gave access directly to the startbox of the second discrimination unit. The startboxes were painted gray, and the alleys were black or white. Each set of triple alleys was mounted on nylon runners so that black and white alleys could be presented in a right-left or left-right order. Hinged, clear Plexiglas covers were mounted over startboxes and alley sections. The black-white alley sets were detachable and were replaced during pretraining by sets of double alleys painted gray.

The presence or absence of chain curtains in either alley served as a second training task dimension. These consisted of nine 6-in. strips of lamp socket pull chains suspended at $1 / 2$-in. intervals from rods placed across the top of the alley. There were two such rod-chain sets, one placed 4 in. from the alley entrance and another 6 in. from the end.

The floor of each alley contained a 1/8-in.-diam hole centered at a point $2 \frac{1}{2}$ in. from the terminus. Metal foodcups ( $1 / 2$ in. deep $\times 1$ in. diam) could be anchored in these holes by means of a stud at the base of the cup. PROCEDURE

All Ss underwent 3 days of pretraining with gray alleys in both discrimination units (hereafter Units 1 and 2). On Day 1, 10 pellets (45 mg) were placed in the foodcups and Ss explored freely for $15 \mathrm{~min}$. Ss in Condition I explored only in Unit 1. These Ss were not allowed access to Unit 2 at any time in the experiment. Ss in Condition II explored in Units 1 

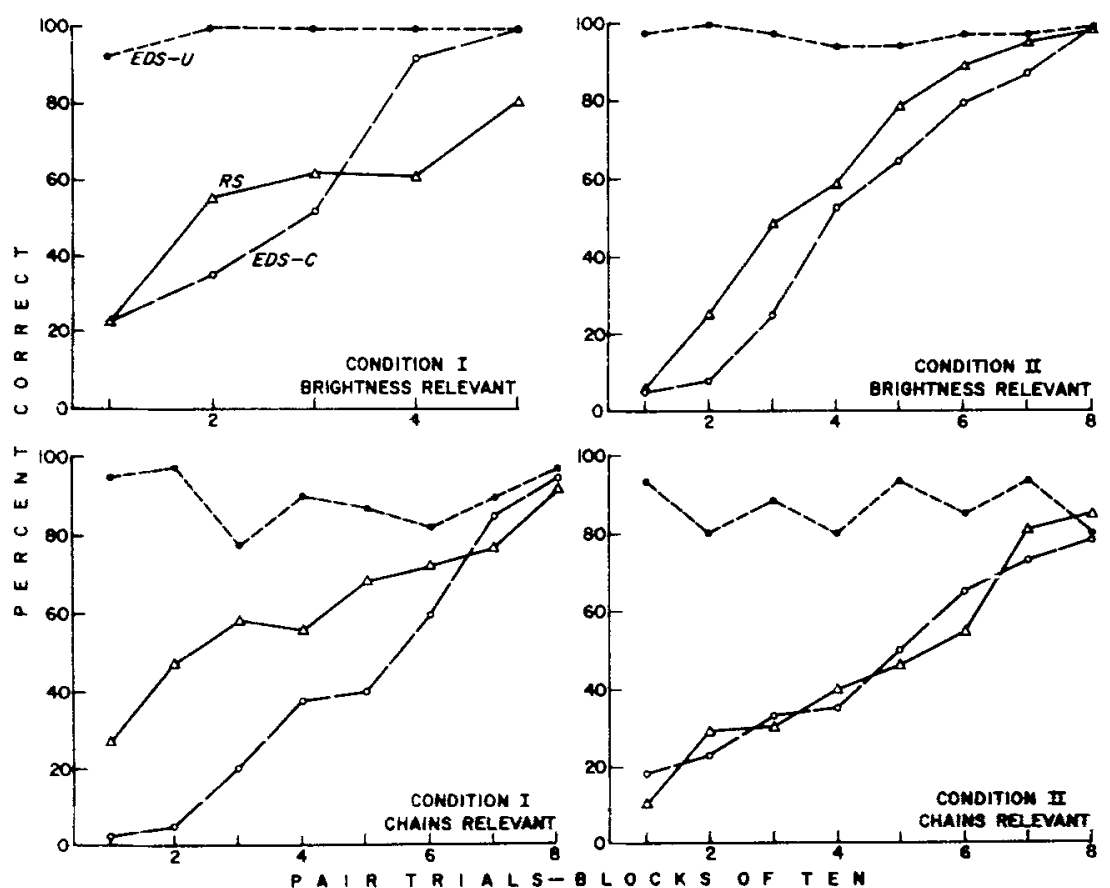

Fig. 1. Learning functions on EDS-U, EDS-C, and RS for each dimension relevant under Conditions I and II.

and 2 and found food in both units. Ss in Condition III explored both units but a foodcup was placed only in Unit 2. For these $\mathrm{Ss}$, the foodcups were removed from Unit 1 throughout the experiment.

On Day 2, Ss were trained to run from startbox to foodcup upon opening of the start doors. Ss in Condition 1 made 20 "runs" through Unit 1 with reinforcement for choice of either alley. As soon as $\mathrm{S}$ consumed the pellets, he was replaced in the startbox for the next run. Ss in Condition II made 10 runs through Units 1 and 2 in succession with reinforcement in both units for choice of either alley. As soon as $S$ received the pellets in Unit 1, the door separating Units 1 and 2 was raised, allowing $S$ access to the start compartment and alleys of Unit 2. When $S$ consumed the pellets in Unit 2, he was replaced in the startbox of Unit 1 for the next run. Ss in Condition III made 10 runs through Units 1 and 2 in succession in the manner of Ss in Condition II, but received reinforcement only in Unit 2 . Note that Conditions I, II, and III had the same number of total choice responses (20). Day 3 of pretraining was conducted in the same manner as Day 2 , but by closing the appropriate alley door prior to the start of each run, each $\mathrm{S}$ was forced to choose a position pattern opposite to that exhibited on his Day 2 training. For all Ss, reinforcement on Days 2 and 3 and during the remainder of the experiment consisted of two $45 \cdot \mathrm{mg}$ food pellets.
During training on the discrimination task, the sequence of events which defined a run in each condition was like that described for pretraining, but receipt of food pellets was now contingent upon choice of the correct member of the constituent stimulus pairs. These consisted of black/chains vs white/no chains and black/no chains vs white/chains. In Condition I, these pairs were presented only in Unit 1 and Ss were reinforced following each correct choice interval was approximately $30 \mathrm{sec}$, during which time $S$ was removed from the apparatus. In Condition II, Ss responded to the stimulus pairs in succession in Units 1 and 2 and were reinforced following choices in each unit. Five seconds after $S$ had nosed into the food dish of Unit 1, E raised the door to Unit 2 , and $S$ invariably proceeded immediately to the choice point. The time between successive choices on the two stimulus pairs in a run, then, was between 5 and $10 \mathrm{sec}$ and averaged about 7 sec. The interrun time was approximately $60 \mathrm{sec}$, during which time $\mathbf{S}$ was removed from the apparatus. In Condition III, Ss also responded to the stimulus pairs in succession in L'nits 1 and 2 but were reinforced only in Unit 2 and only if they had chosen the correct pair member in both units. Food reward was placed in Unit 2 only if $S$ had chosen correctly in Unit 1 , but $\mathrm{E}$ went through identical placing motions on every run regardless of the nature of S's choice on Unit 1 . These motions response. The interrun (or interchoice) were made during the forced 5 -sec interunit delay described for Condition II. The within-run and interrun intervals in Condition III were the same as those of Condition II.

All Ss received 10 presentations of each stimulus pair per day. In Conditions II and III, Units 1 and 2 always contained the two different stimulus pairs on any run, but the location of a particular pair varied randomly from one run to the next. The order in which the pairs were presented over the entire training sequence and the sequence of left vs right position of pair members [determined by a Fellows (1967) secuence] were identical for Conditions I, II, and III. Thus, Conditions II and III provide different degrees of organization of the constituent pairs but within the same overall presentation sequence as Condition I. Ss in II and III respond to the constituent pairs in temporally and spatially defined runs, and $S s$ in III must in addition exhibit common response across the pairs in order to receive reinforcement. Condition $I$ training is that of the conventional discrimination shift experiment and approximated the conditions of Kelleher's (1956) study.

Black, white, chains present, and chains absent were used equally often as the positive cue within all conditions. Ss in Conditions I and II were trained to a criterion of at least 18 correct choices on each of 2 successive days, and $S s$ in Condition III, to a criterion of at least nine correct runs (and thus 18 correct choices) on each of 2 successive days. On the day following attainment of criterion, one-half of each group received an $R$ shift and one-half, an ED shift. Shift training was identical in all respects to that of the initial discrimination, and was continued to the aforementioned criteria.

The Ss were distributed in groups of 16,16 , and 8 in Conditions I, II, and III, respectively.

\section{RESULTS}

The data were to be collected in two replications, each consisting of eight Ss in each condition. This plan was completed for Conditions I and II but proved infeasible for Condition III due to the inordinate difficulty of that task. Consequently, the data of Conditions I and II will first be analyzed, followed by consideration of those Condition III Ss of the initial group of eight who completed training within the time span of the experiment.

Factorial analyses of variance involving Dimension by Shift Assignment by Condition (I vs II) were applied to $\sqrt{\mathrm{x}+.5}$ transformed trials and errors to criterion in the initial discrimination and in the shift 

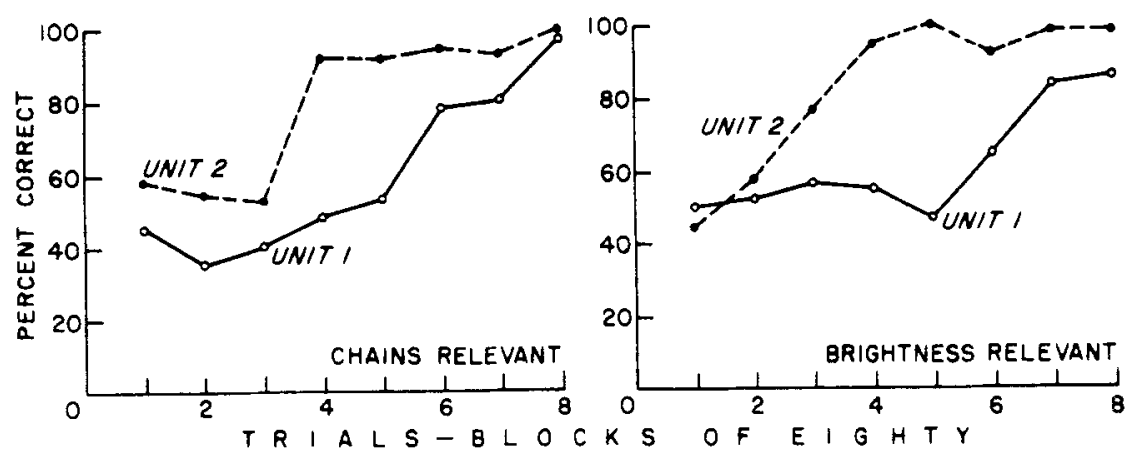

Fig. 2. Learning functions on Units 1 and 2 in Condition III for an S trained with chains relevant and for an $S$ trained with brightness relevant.

problems. Learning of the initial discrimination was found to proceed more slowly in Condition II than in Condition $I$ ( $F=12.2$ and 10.1 for the trial and error measures, respectively, and $p<.005$ for both measures). No other $F$ value approached significance in the analyses of the original learning scores.

Analysis of the error scores in the shift discriminations showed that learning was easier with brightness relevant than with chain cues relevant $(\mathrm{F}=17.2, \mathrm{p}<.001)$, that learning was more difficult under Condition II than under Condition $I(F=8.0, p<.01)$, and that ED shift was accomplished more easily than $R$ shift ( $F=14.3$, $p<.001)$. None of the $F$ values for interaction effects approached significance. Analysis of the trial scores yielded identical outcomes, except that the shift difference did not attain significance.

The data of major concern in the experiment are shown in Fig. 1, which depicts shift learning in each major condition in terms of performance on individual problem units. In interpreting these functions, it should be recalled that an ED shift involves reversal of the stimulus-reward relations on one of the stimulus pairs, the other pair retaining the reward relations that obtained in the initial discrimination. For $\mathrm{R}$ shift, of course, stimulus-reward relations are reversed for both of the pairs. The graphs show the group learning functions for the changed pair in ED shift (EDS-C) and the unchanged pair (EDS-U), along with the averaged functions for the two pairs of reversal shift (RS).

Considering ED shifts with brightness relevant, the EDS-C and EDS- $U$ functions clearly indicate that the rats learned the constituent pairs of this task as independent subproblems in Conditions I and II. Performance on EDS-U is virtually error-free in both conditions, while the error rate on EDS-C resembles that of Ss undergoing complete reversal learning. Among Ss who learned ED shift with chain cues relevant, there is a somewhat higher error rate on EDS-U. Nevertheless, independence in subproblem learning by these Ss is also clearly evidenced in Conditions I and II in that performance on the unchanged pair is at or very near criterion ( $90 \%$ correct) throughout ED learning, while there is a slow, gradual acquisition of correct response to the changed pair. Conditions I and II do not differ in the degree of independence manifested in learning the constituent pairs of the ED problem. The mean proportion of total ED shift errors occurring on the unchanged pair was .13 among Condition I Ss and .14 for Ss of Condition II, $\mathrm{t}=.17, \mathrm{df}=14$.

Only six of the eight Ss trained in Condition III mastered the initial discrimination within the 1,000-trial limit set. Nevertheless, the manner in which these Ss achieved solution of the initial discrimination is of interest to the issue of independent subproblem learning. It will be recalled that in this condition reinforcement was contingent upon choice of the relevant cue in the successive pairs of Units 1 and 2.On this basis, it might be Expected that correct responding in the two discrimination units would be highly correlated. Instead, these Ss generally learned to choose correctly in Unit 2 many trials in advance of the appearance of correct responding in Unit I. Figure 2 shows this learning pattern as it appeared in its clearest form under each condition of dimension relevancy.

The shift data of Condition III Ss resembles that observed under Conditions I and II. Three Ss received ED shift and, within dimensions, each made fewer errors to criterion than three $S s$ given $R$ shift. As seen in Fig. 3, the pattern of subproblem learning in the $\mathrm{ED}$ shift $\mathrm{Ss}$ was similar to those seen in Conditions I and II.

\section{DISCUSSION}

Subproblem analysis of shift learning in the rat revealed that relearning during ED shift was largely confined to the changed element of the problem. Essentially, ED shift posed one reversal subproblem for the rats and $R$ shift posed two reversal subproblems. The same pattern of subproblem learning was exhibited by turtles in similar tasks, and this species, like rats, found ED easier than $R$ shift (Graf \& Tighe, 1971). The subproblem and shift performance of both species should be contrasted to that reported by Tighe et al (1971) for a paradigmatically and structurally similar ED shift by 10-year-old

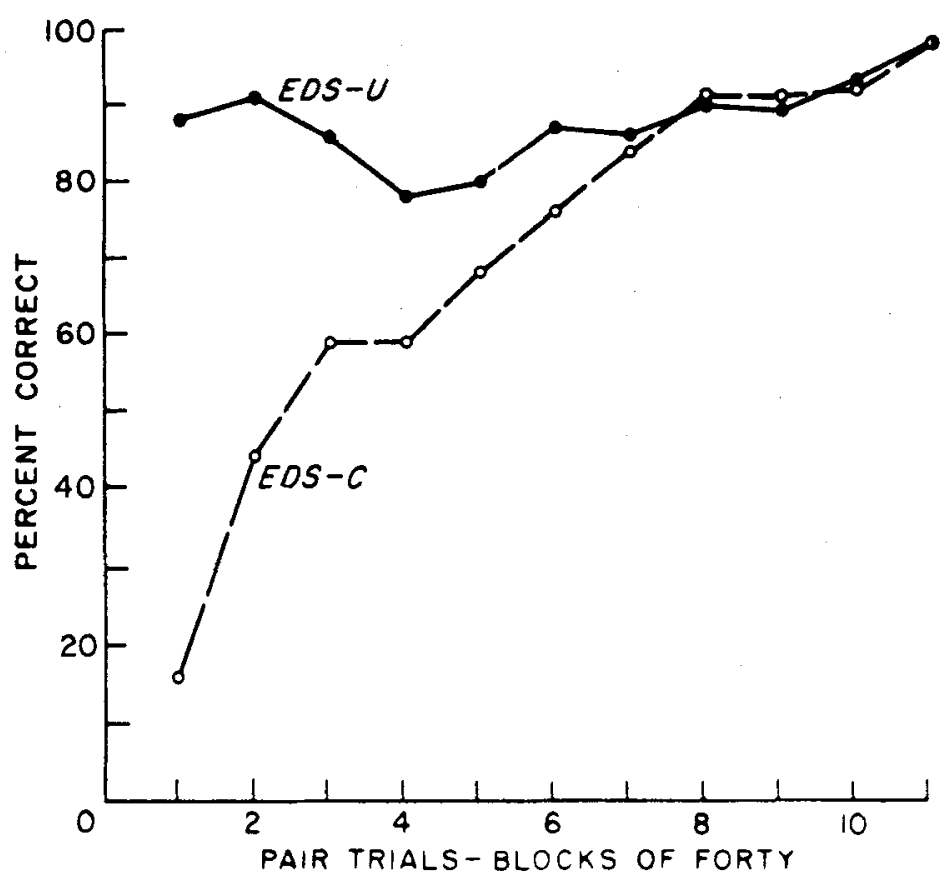

Fig. 3. Learning functions on EDS-U and EDS-C under Condition III. 


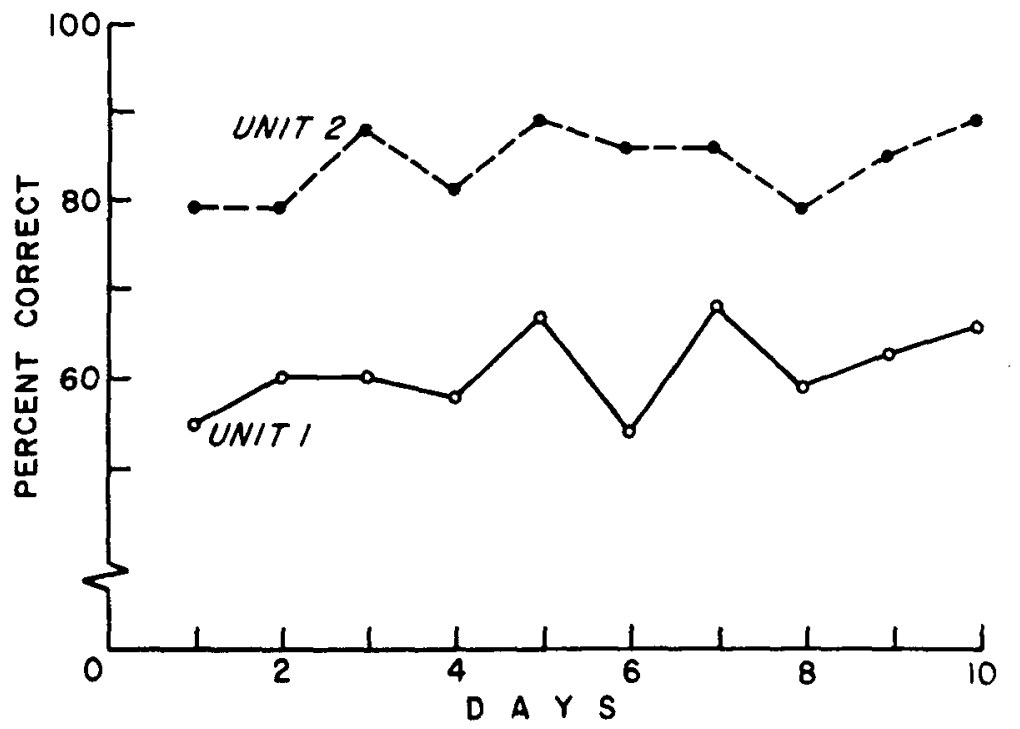

Fig. 4. Backward learning curves on Units 1 and 2 in Condition $I I$ over the last 10 precriterion days. As Ss attain criterion in this interval, they are excluded from determination of the learning curves.

children. The children's EDS-U and EDS-C learning functions overlapped throughout shift, and the unitary manner in which these elements were learned was manifest at the outset of shift. Sixty-three percent of these Ss reversed their choice on the first postshift exposure to EDS-U after experiencing nonreward on EDS-C. That is, nonreward on EDS-C caused these $\mathrm{Ss}$ to change response on EDS-U, even though they had never experienced nonreward on this pair. Such "spontaneous reversal" of choice was never observed in the present experiment or in the performance of the turtles. The children found ED shift significantly more difficult than R shift.

This pattern of findings bears differentially on the major theories of discrimination shift learning. On the one hand, the pattern fits Kendler \& Kendler's (1962) formulation which assumes that infrahumans discriminate on the basis of single-stage associations in the manner indicated by Spence, while the mature human discriminates on the basis of dimension-specific mediating representational responses. An application of a computer simulation of Spence's model (Wolford \& Bower, 1969) to this situation showed that marked degrees of independence in subproblem learning, similar to that exhibited herein, is not an unlikely outcome of Spence's learning process. 1

On the other hand, the virtually complete independence in subproblem learning observed in animal learning is counter to attentional theory (e.g., Sutherland, 1959; Zeaman \& House, $1963)$, which assumes that dimension-specific attentional responses direct discrimination learning in all $S s$ and that attentional response to the relevant dimension is prerequisite to learning of the correct choice response. If choice responses on each pair of the shift task are linked to an attentional response common to the pairs, then weakening of that attentional response through nonreinforcement on EDS-C should be reflected in disruption of performance on EDS-U. But the functions in Fig. 1 provide no evidence that transfer learning involved extinction and acquisition of dimensional observing responses (i.e., a redirection of attention to dimensions) prior to

The data on subproblem independence in animals do not, of course, deny that dimensional attention responses may operate in the discrimination learning of these Ss (cf. Shepp \& Eimas, 1964), nor strictly speaking do these data bear on the role of such responses during the original learning phase of the present experiment. But the present data do indicate that dimensional control of discriminative response in such transfer problems by animals may be of a much more limited and conditional nature than generally supposed.

Although Condition I produced the expected pattern of independent subproblem learning, Condition II proved ineffective as a means of modifying that pattern and, moreover, significantly slowed pre- and postshift learning. A possible interpretation of this outcome, suggested by Estes's analysis (1970) of opposing intertrial solution of the ED shift problem. interval processes, is that the frequent brief interpair intervals within the runs of Condition II were of little or no benefit in facilitating detection of the common cue-reward relation across pairs (the intended effect), and that the dominant effect of the brief intervals was interference with consolidation of the temporary associations established to the preceding pair.

Support for this view is seen in Fig. 4, which shows group "backward learning" functions on Units 1 and 2 over the last 10 precriterion days. Day 10 in this figure shows the mean percent correct over each S's last precriterion day, etc. It will be recalled that a very brief interval separates successive choices on Units 1 and 2 , while there is a relatively long interchoice interval following response in Unit 2. Figure 4 shows that learning in Unit 2 is clearly superior to that in Unit 1 , as suggested by an anticonsolidation hypothesis. But the differential in learning in the two units becomes particularly interesting when it is considered that the stimulus pairs appear equally often in each unit. Development of correct choice on the pairs, then, is not independent of their context, and this outcome can be taken as further indication of the concrete nature of the learning process in these subjects.

In regard to learning under Condition III, the solution pattern illustrated in Fig. 2 indicates that the Ss were insensitive to the contingency between choice in Unit 1 and reward in Unit 2. In interpreting this solution pattern, it should be noted that while these Ss were performing at a chance level on each stimulus pair as these pairs appeared in Unit 1 , they were concurrently choosing the correct cue on the same pairs during their sustained criterion performance on Unit 2-and for only $50 \%$ reinforcement. These considerations suggest the operation of a relatively simple learning mechanism as the basis of solution of the Condition III problem, rather than the integration of information over successive choices on the constituent pairs. For example, the solution pattern might be accounted for in terms of a gradual accrual of secondary reinforcement properties by cues in the second unit. Whatever mechanisms are involved, it is clear that Condition III training as implemented herein is an infeasible, and probably an ineffective, means of reducing independence in the learning of the ED shift subproblems.

\section{REFERENCES}

ESTES, W. K. Learning theory and mental development. New York: Academic Press, 1970. 
FELLOWS, B. J. Chance stimulus sequences for discrimination tasks. Psychological Bulletin, 1967, 67, 87-92.

GRAF, V., \& TIGHE, T. Subproblem analysis of discrimination shift learning in the turtle (Chrysemys picta picta). Psychonomic Science, 1971, 25, 257-259.

KELLEHER, R. I. Discrimination learning as a function of reversal and nonreversal shifts. Journal of Experimental Psychology, 1956,51, 379-384.

KENDLER, H. H., \& KENDLER, T. S. Vertical and horizontal processes in problem solving. Psychological Review, $1962,69,1-16$.

KENDLER, H. H. Environmental and cognitive control of behavior. American Psy chologist, 1971, 26, 962-973.

LEARY, R. W. "Spontaneous reversal" in the serial-discrimination reversal learning of monkeys. Canadian Journal of Psychology, 1962, 16, 228-233.

SHEPP, B. E., \& EIMAS

Intradimensional and extradimensional shifts in the rat. Journal of Comparative \& Physiological Psychology, 1964, 57, 357-361.

SUTHERLAND, N. S. Stimulus analysing mechanisms. In Proceedings of $a$ symposium on the mechanisation of thought processes. Vol. 2. London: Her Majesty's Stationery Office, 1959.
TIGHE, T. J., GLICK, J., \& COLE, M. $S$ u b p r o b l e m a nalysis o discrimination-shift learning. Psychonomic Science, 1971, 24, 159-160.

WOLFORD, G. \& BOWER, G. H. Continuity theory revisited: Rejected for the wrong reasons? Psychological Review, $1969,76,515-518$.

ZEAMAN, D., \& HOUSE, B. J. The role of attention in retardate discrimination learning. In N. R. Ellis (Ed.), Handbook of mental deficiency. New York: McGraw-Hill, 1963. Pp. 159-223.

NOTE

1. Program provided by George Wolford. 